\title{
Preocupação com a forma do corpo de graduandos de Farmácia-Bioquímica
}

\author{
Body shape concern of the graduate students of Pharmacy-Biochemistry
}

Bianca Gonzalez Martins', Wanderson Roberto Silva², Juliana Alvares Duarte Bonini Campos²

\section{RESUMO}

Objetivo: Identificar a preocupação com a forma do corpo de estudantes de Farmácia-Bioquímica e sua relação com variáveis sociais e laborais e com o estado nutricional. Métodos: Participaram 346 discentes com média de idade de 20,2 (DP = 2,4) anos, sendo $278(80,3 \%)$ do sexo feminino. Utilizou-se o Body Shape Questionnaire (BSQ). As validades fatorial e convergente e a consistência interna (a) do BSQ foram estimadas. Utilizaram-se como índices de ajustamento o qui-quadrado pelos graus de liberdade $\left(\chi^{2} / \mathrm{gl}\right)$, o Comparative Fit Index (CFI), o Normed Fit Index (NFI) e o Root Mean Square Error of Approximation (RMSEA). O escore médio de preocupação com a forma do corpo foi obtido por meio de algoritmo gerado na análise fatorial confirmatória. Para comparar os escores médios segundo as variáveis de interesse, utilizou-se Análise de Variância (ANOVA). Resultados: $O$ BSQ apresentou, para a amostra de estudo, adequada validade $\left(\chi^{2} / \mathrm{gl}=3,29 ; \mathrm{CFI}=0,87, \mathrm{NFI}=0,82\right.$, RMSEA $\left.=0,08\right)$ e confiabilidade $(\alpha=0,97)$ após ajustamento. Verificou-se que as mulheres $(p<0,001)$ apresentaram maior preocupação com a forma do corpo que os homens. Além disso, os estudantes que avaliaram o curso como pior que as expectativas iniciais ( $p=0,048)$, que consomem medicamentos por causa dos estudos ( $p<0,001$ ), que já pensaram em desistir do curso ( $p=$ $0,002)$ e foram classificados com sobrepeso/obesidade $(p<0,001)$ também apresentaram alta

\section{Palavras-chave}

Imagem corporal, estudantes de farmácia, questionários. preocupação com a forma do corpo. Conclusão: As varáveis sexo, avaliação em relação ao curso, ingestão de medicamentos por causa dos estudos, pensamento em desistir do curso e o estado nutricional apresentaram relação significativa com a preocupação com a forma do corpo entre os estudantes.

\section{ABSTRACT}

Objective: To determine the body shape concern among pharmacy-biochemistry students and its association with several social and labor variables and nutritional status. Methods: $\mathrm{A}$ total of 346 students ( $80.3 \%$ female) with mean age $20.2(S D=2.4)$ years were asked to answer the Body Shape Questionnaire (BSQ). The factorial and convergent validities and the internal consistencies ( $\mathrm{a}$ ) of the BSQ were calculated. The goodness-of-fit indices, the chi-square by degree of freedom ratio $\left(\chi^{2} / \mathrm{df}\right)$, the comparative fit index (CFI), the normed fit index (NFI), and the root mean square error of approximation (RMSEA) were also used for data analysis. The

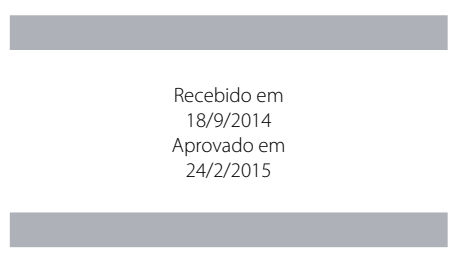

DOI: $10.1590 / 0047-2085000000054$
1 Universidade Estadual Paulista (Unesp), Faculdade de Ciências Farmacêuticas de Araraquara, Curso de Graduação em Farmácia-Bioquímica. 2 Unesp, Faculdade de Ciências Farmacêuticas de Araraquara, Departamento de Alimentos e Nutrição.

Endereço para correspondência: Juliana Alvares Duarte Bonini Campos Universidade Estadual Paulista (Unesp), Faculdade de Ciências Farmacêuticas de Araraquara, Departamento de Alimentos e Nutrição

Rodovia Araraquara-Jaú, km 01 14801-902 - Araraquara, SP, Brasil Telefone: (16) 3301-6358

E-mail: jucampos@fcfar.unesp.br 


\section{Keywords}

Body image, pharmacy

students, questionnaires. mean score of body shape concern was estimated using an algorithm generated from the confirmatory factor analysis. Analysis of variance (ANOVA) was used to compare the mean scores with variables of interest. Results: The BSQ presented adequate validity $\left(\chi^{2} / \mathrm{df}=3.29\right.$; $\mathrm{CFI}=.87, \mathrm{NFI}=.82, \mathrm{RMSEA}=.08)$, and reliability $(\mathrm{a}=.97)$ after fit. Female students showed more body shape concern than male students $(p<.001)$. Furthermore, the students who rated the course as dissatisfactory relative to their initial expectation $(p=.048)$, consumed drugs under academic pressure $(p<.001)$, thought about quitting the course $(p=.002)$, and were overweight/obese $(p<.001)$ also presented greater body shape concern. Conclusion: The variables gender, dissatisfaction with the course, drug intake due to academic pressure, desire of quitting the course, and the nutritional status were significantly associated with the body shape concern among the participating students.

\section{INTRODUÇÃO}

A representação mental que um indivíduo faz do seu próprio corpo é denominada imagem corporal. A construção desse conceito envolve aspectos afetivos, sociais e físicos que se manifestam durante toda a vida, sofrendo constantes transformações de acordo com as experiências vividas ${ }^{1-3}$. A forma de lidar com a imagem corporal é individual e, geralmente, é norteada por parâmetros impostos pela sociedade, pela cultura vigente e pelo ambiente no qual o indivíduo está inserido ${ }^{4}$.

A imagem corporal é construída com base em diversos aspectos como a preocupação com a forma do corpo e com peso corporal, a estrutura muscular e a satisfação com a aparência física ${ }^{3-5}$.

A preocupação com a forma do corpo é um dos aspectos da imagem corporal mais investigados ${ }^{6-9} \mathrm{e}$ tem relação direta com fatores psicológicos, cognitivos e comportamentais, podendo se manifestar em qualquer indivíduo vulnerável ${ }^{10}$. É importante destacar que alguns estudos ${ }^{11-13}$ tratam a preocupação com a forma do corpo como sinônimo de imagem corporal, o que não pode ser considerado adequado, uma vez que essa se refere apenas a um aspecto desse conceito ${ }^{14}$.

$\mathrm{Na}$ busca de mensurar aspectos relacionados à imagem corporal, foram desenvolvidos vários instrumentos de medida, entre os quais se podem destacar o Eating Disorder Inventory $(\mathrm{EDI})^{15}$, a Escala de Silhuetas ${ }^{16}$, o Body Image Avoidance Questionnaire (BIAQ) $)^{17}$ e o Body Shape Questionnaire (BSQ) ${ }^{18}$.

O BSQ é um dos instrumentos mais utilizados na literatura, tanto em contexto brasileiro ${ }^{10,11,14,19-21}$ quanto internacional $\left.\right|^{6,22-25}$, para estimar a preocupação com a forma do corpo em indivíduos normativos ou clínicos. O BSQ foi desenvolvido por Cooper et al. ${ }^{18}$ para a população inglesa e possui 34 itens, de autopreenchimento, com respostas do tipo Likert de seis pontos, que avaliam o nível de preocupação que o indivíduo tem em relação à forma do seu corpo.

Estudos que administraram o BSQ ${ }^{12,19,21,26}$ indicam que os jovens, principalmente na fase da adolescência e início da vida adulta, são os mais acometidos pelas alterações relacionadas à imagem corporal, o que os torna mais vulneráveis às questões ambientais, sociais, culturais e estéticas ${ }^{12}$. Sepulveda et al. ${ }^{27}$ destacam que o ingresso dos jovens em ambiente universitário promove mudanças de paradigmas sociais repentinos, o que pode aumentar a tensão e a ansiedade e favorecer o desenvolvimento de alterações referentes à percepção da própria aparência física. A literatura acrescenta, ainda, que variáveis como sexo9 ${ }^{9}$, nível econômico ${ }^{28}$, estado nutriciona ${ }^{21}$ e presença de atividade laboral concomitante aos estudos ${ }^{29}$ também podem interferir na preocupação de um indivíduo quanto à forma do seu corpo.

Outro aspecto importante destacado na literatura ${ }^{11,26,30-32}$ é a investigação de fatores relacionados à imagem corporal em discentes de cursos ligados à área da saúde, uma vez que eles serão profissionais cuja atuação estará diretamente relacionada à área de promoção da saúde e, portanto, deveriam servir de exemplo e ter estilo de vida saudável.

Investigar aspectos relacionados à preocupação com a forma do corpo pode auxiliar na detecção precoce de alterações relacionadas à imagem corporal dos indivíduos, o que pode contribuir para a elaboração de estratégias educativo-preventivas que possam minimizar a ocorrência de danos futuros. Nesse contexto, conduziu-se este estudo com o objetivo de identificar a preocupação com a forma do corpo e sua relação com variáveis sociodemográficas, laborais e com o estado nutricional de estudantes do curso de graduação em Farmácia-Bioquímica de uma universidade pública do estado de São Paulo.

\section{MÉTODOS}

\section{Desenho de estudo e delineamento amostral}

Trata-se de estudo observacional do tipo transversal, com delineamento amostral não probabilístico. Foram convidados a participar todos os estudantes matriculados no ano de 2013 ( $n=430$ ) no curso de graduação em Farmácia-Bioquímica da Faculdade de Ciências Farmacêuticas da Universidade Estadual Paulista (Unesp), campus de Araraquara. Cabe 
esclarecer que os estudantes do último ano não foram incluídos no estudo por estarem realizando atividades em ambiente externo à universidade, o que inviabilizou o acesso dos pesquisadores a eles.

O tamanho amostral mínimo foi estimado considerando a necessidade de oito respondentes por item do instrumento (BSQ), o que resultou em 272 participantes. Contudo, considerou-se, ainda, uma taxa de perda de 15\%, corrigindo o tamanho amostral para 320 estudantes $^{33}$.

\section{Variáveis de estudo e instrumento de medida}

Para caracterização da amostra, foram levantadas informações como sexo, idade, ano e período do curso. Foram coletadas, também, informações sobre expectativa e desempenho no curso, uso de medicação por causa dos estudos, pensamento de desistir do curso, presença de atividade laboral concomitante aos estudos, moradia, ordem de preferência pelo curso no vestibular e necessidade de recorrer a professores particulares.

Para cômputo do índice de massa corporal (IMC) $\left(\mathrm{kg} / \mathrm{m}^{2}\right)$, o peso $(\mathrm{kg})$ e a altura $(\mathrm{m})$ referidos foram investigados para posterior classificação do estado nutricional dos participantes, segundo proposta da Organização Mundial de Saúde ${ }^{34}$. O nível econômico foi estimado utilizando-se o Critério Bra$\mathrm{sil}^{35}$.

Para estimar a preocupação com a forma do corpo dos estudantes, foi utilizado o Body Shape Questionnaire (BSQ). A versão em português utilizada foi a apresentada por Di Pietro e Silveira ${ }^{20}$. Entretanto, nesse trabalho, a estrutura fatorial do BSQ utilizada seguiu a proposta original de Cooper et al..$^{36}$, cujas validade e confiabilidade em estudantes universitárias brasileiras foram estimadas e atestadas por Silva et al..14.

\section{Procedimentos e aspectos éticos}

Os estudantes preencheram a versão completa do BSQ e um questionário sociodemográfico em sala de aula, com a presença do professor responsável pela disciplina, que previamente autorizou, por escrito, a aplicação deles. Antes do preenchimento, os estudantes foram informados sobre o objetivo da pesquisa e sobre a participação voluntária. Os questionários foram identificados apenas com um código numérico, preservando o anonimato dos participantes.

A realização do estudo foi aprovada pelo Comitê de Ética em Pesquisa em Seres Humanos da Faculdade de Ciências Farmacêuticas da Unesp, campus de Araraquara (protocolo 11/2012).

\section{Validade e confiabilidade do BSQ na amostra}

A validade de construto foi estimada por meio da validade fatorial e convergente. A validade fatorial foi avaliada por meio de análise fatorial confirmatória (AFC). Foram utilizados os índices de avaliação da qualidade de ajustamento, razão de qui-quadrado pelos graus de liberdade $\left(\chi^{2} / g\right)$ ), Compara- tive Fit Index (CFI), Normed Fit Index (NFI) e o Root Mean Square Error of Approximation (RMSEA) ${ }^{37}$. O ajustamento foi considerado adequado quando $\lambda>0,40, \chi^{2} / \mathrm{gl} \leq 3,0, \mathrm{CFI} \geq 0,90, \mathrm{NFI} \geq$ 0,80 e RMSEA $\leq 0,10^{37}$. A validade convergente foi aferida por meio da Variância Extraída Média (VEM) e da Confiabilidade Composta (CC) e foram consideradas adequadas VEM $\geq 0,50$ e CC $\geq 0,70$, respectivamente ${ }^{38}$.

A consistência interna foi calculada utilizando-se o coeficiente alfa de Cronbach padronizado $(\alpha)$ e considerada adequada quando $\alpha \geq 0,70^{39}$.

O escore médio de preocupação com a forma do corpo dos estudantes foi calculado a partir da matriz dos pesos de regressão obtida pela matriz de covariância entre as variáveis manifestas e latentes do modelo fatorial do $\mathrm{BSQ}^{40}$.

\section{Análise estatística}

A Análise de Variância (ANOVA) foi conduzida para comparar os escores médios de preocupação com a forma do corpo dos estudantes segundo as variáveis de interesse. Os escores médios foram obtidos a partir do algoritmo estimado pela matriz de regressão. Os pressupostos de normalidade e homocedasticidade foram verificados e atendidos por meio dos testes de Shapiro-Wilk ( $p=0,05-0,337$ ) e Levene ( $p=$ 0,05-0,716), respectivamente. Quando da detecção de diferenças significativas, utilizou-se o pós-teste de Tukey para comparações múltiplas. Para tomada de decisão, foi adotado nível de significância de 5\%.

Os programas IBM SPSS Statistics (v.21, SPSS An IMB Company, Chicago, IL) e AMOS 21.0 (IBM SPSS Inc, Chicago, IL) foram utilizados para realização das análises.

\section{RESULTADOS}

Participaram do estudo 346 estudantes, com média de idade de 20,2 ( $(D P=2,4)$ anos, sendo 80,3\% do sexo feminino. A caracterização sociodemográfica dos participantes apresenta-se na tabela 1.

Observa-se que a maior parte dos estudantes concentra-se no primeiro ano e no período integral do curso. Também se nota que a maioria nunca recorreu a professores particulares, escolheu o curso de Farmácia-Bioquímica como primeira opção no vestibular, nunca necessitou tomar medicamentos por causa dos estudos, não possui atividade laboral concomitante aos estudos, apresenta suas expectativas iniciais em relação ao curso atendidas e considera-se como bons alunos. O IMC médio dos estudantes foi de 22,6 (DP = 3,8) $\mathrm{kg} / \mathrm{m}^{2}$. Quanto ao estado nutricional, a maior parte dos estudantes foi classificada como eutrófica.

Na tabela 2 apresenta-se a distribuição de frequências das respostas dadas aos itens do BSQ. 
Tabela 1. Escore médio \pm desvio-padrão da preocupação com a forma do corpo segundo as variáveis de interesse (São Paulo, 2013)

\begin{tabular}{|c|c|c|c|c|c|}
\hline Característica & $\mathrm{n}(\%)$ & $\begin{array}{c}\text { Média } \pm \\
\text { desvio-padrão }\end{array}$ & $\mathrm{p}$ & $\eta_{p}^{2}$ & $\pi$ \\
\hline \multicolumn{6}{|l|}{ Sexo } \\
\hline Masculino & $68(19,7)$ & $1,00 \pm 0,43$ & $<0,001^{*}$ & 0,052 & 0,991 \\
\hline Feminino & $278(80,3)$ & $1,31 \pm 0,56$ & & & \\
\hline \multicolumn{6}{|l|}{ Ano do curso } \\
\hline Primeiro & $164(47,5)$ & $1,25 \pm 0,53$ & 0,549 & 0,009 & 0,245 \\
\hline Segundo & $73(21,2)$ & $1,27 \pm 0,56$ & & & \\
\hline Terceiro & $41(11,9)$ & $1,24 \pm 0,56$ & & & \\
\hline Quarto & $57(16,5)$ & $1,32 \pm 0,59$ & & & \\
\hline Quinto e sexto & $10(2,9)$ & $1,00 \pm 0,49$ & & & \\
\hline \multicolumn{6}{|l|}{ Professores particulares } \\
\hline Sim & $88(25,6)$ & $1,26 \pm 0,55$ & 0,449 & 0,002 & 0,118 \\
\hline Não & $256(74,4)$ & $1,20 \pm 0,52$ & & & \\
\hline \multicolumn{6}{|l|}{ Ordem de preferência pelo curso } \\
\hline Primeira opção & $278(81,0)$ & $1,23 \pm 0,55$ & 0,062 & 0,010 & 0,464 \\
\hline Outra & $65(19,0)$ & $1,37 \pm 0,51$ & & & \\
\hline \multicolumn{6}{|c|}{ Em relação às suas expectativas iniciais, este curso é: } \\
\hline Melhor & $165(47,7)$ & $1,19 \pm 0,50^{\mathrm{a}}$ & $0,048^{*}$ & 0,018 & 0,589 \\
\hline Igual & $117(33,8)$ & $1,27 \pm 0,55^{\mathrm{a}, \mathrm{b}}$ & & & \\
\hline Pior & $64(18,5)$ & $1,39 \pm 0,62^{b}$ & & & \\
\hline \multicolumn{6}{|l|}{ Desempenho no curso } \\
\hline Bom & $193(56,1)$ & $1,21 \pm 0,51$ & 0,118 & 0,007 & 0,346 \\
\hline Ruim & $151(43,9)$ & $1,30 \pm 0,58$ & & & \\
\hline \multicolumn{6}{|l|}{ Com quem você vive } \\
\hline Sozinho & $59(17,4)$ & $1,29 \pm 0,53$ & 0,588 & 0,003 & 0,138 \\
\hline Família & $65(19,1)$ & $1,28 \pm 0,51$ & & & \\
\hline Amigos, colegas & $216(63,5)$ & $1,22 \pm 0,55$ & & & \\
\hline \multicolumn{6}{|c|}{ Você já precisou tomar algum tipo de medicação por causa dos estudos? } \\
\hline Não & $230(66,9)$ & $1,17 \pm 0,49$ & $<0,001^{*}$ & 0,042 & 0,973 \\
\hline Sim & $144(33,1)$ & $1,41 \pm 0,62$ & & & \\
\hline \multicolumn{6}{|l|}{ Você já pensou em desistir do curso? } \\
\hline Não & $181(52,5)$ & $1,17 \pm 0,50$ & $0,002^{*}$ & 0,027 & 0,863 \\
\hline Sim & $164(47,6)$ & $1,35 \pm 0,58$ & & & \\
\hline \multicolumn{6}{|l|}{ Atividade laboral concomitante aos estudos } \\
\hline Não & $308(89,8)$ & $1,25 \pm 0,55$ & 0,601 & 0,001 & 0,082 \\
\hline Sim & $35(10,2)$ & $1,30 \pm 0,51$ & & & \\
\hline \multicolumn{6}{|l|}{ Estado nutricional } \\
\hline Baixo peso & $8(3,1)$ & $0,79 \pm 0,27^{a}$ & $<0,001^{*}$ & 0,144 & 1,000 \\
\hline Eutrofia & $202(78,6)$ & $1,17 \pm 0,49^{b}$ & & & \\
\hline Sobrepeso/obesidade & $47(18,3)$ & $1,64 \pm 0,57^{c}$ & & & \\
\hline \multicolumn{6}{|l|}{ Nível econômico (renda mensal média) } \\
\hline Classe A (R\$ 8.295,00 a $R \$ 11.480,00)$ & $150(43,9)$ & $1,23 \pm 0,54$ & 0,324 & 0,007 & 0,248 \\
\hline Classe $B(R \$ 2.656,00$ a $R \$ 4.754,00)$ & $157(45,9)$ & $1,29 \pm 0,54$ & & & \\
\hline Classe C (R\$ 962,00 a R\$1.459,00) & $35(10,2)$ & $1,16 \pm 0,60$ & & & \\
\hline
\end{tabular}

* Diferença estatística significativa para $a=0,05$; a,bc letras iguais indicam similaridade estatística. 
Nota-se que a maioria dos itens foi preenchida e que alguns concentraram as respostas em uma opção, por exemplo, grande parte dos estudantes indicou a opção "nunca" nos itens 26 e 32, o que comprometeu a sensibilidade psicométrica desses itens.

A análise fatorial confirmatória do BSQ revelou ajustamento insatisfatório à amostra $\left(\chi^{2} / \mathrm{gl}=4,04, \mathrm{CFI}=0,81\right.$, NFI $=0,76$, RMSEA $=0,09$ ) e baixos pesos fatoriais nos itens 26 $(\boldsymbol{\lambda}=0,35)$ e $32(\boldsymbol{\lambda}=0,33)$. Assim, realizou-se o refinamento do modelo excluindo-se esses itens. Na figura 1 apresenta-se o modelo refinado ajustado do BSQ para a amostra de estudo.

No modelo refinado, foram inseridas correlações entre os erros dos itens 2 e 21, 3 e 10, 6 e 17, 7 e 18, 10 e 27, 24 e 28 e 28 e 30. Após o refinamento e a inserção das correlações, o BSQ apresentou ajustamento satisfatório à amostra $\left(\chi^{2} / g l=\right.$ $3,29, \mathrm{CFI}=0,87, \mathrm{NFI}=0,82$, RMSEA $=0,08$ ).

Tabela 2. Distribuição de frequências [n(\%)] de respostas dadas ao Body Shape Questionnaire (BSQ) pelos discentes participantes da amostra (São Paulo, 2013)

\begin{tabular}{|c|c|c|c|c|c|c|}
\hline \multirow{2}{*}{ Item } & \multicolumn{6}{|c|}{$\mathrm{n}(\%)$} \\
\hline & Nunca & Raramente & Às vezes & Frequentemente & Muito frequentemente & Sempre \\
\hline It 1 & $58(16,8)$ & $65(18,8)$ & $123(35,7)$ & $53(15,4)$ & $28(8,1)$ & $18(5,2)$ \\
\hline It 2 & $80(23,1)$ & $54(15,6)$ & $67(19,4)$ & $46(13,3)$ & $48(13,9)$ & $51(14,7)$ \\
\hline It 3 & $158(45,7)$ & $48(13,9)$ & $54(15,6)$ & $29(8,4)$ & $22(6,4)$ & $35(10,1)$ \\
\hline It 4 & $60(17,4)$ & $37(10,8)$ & $82(23,8)$ & $52(15,1)$ & $52(15,1)$ & $61(17,7)$ \\
\hline It 5 & $48(13,9)$ & $54(15,6)$ & $99(28,6)$ & $57(16,5)$ & $50(14,5)$ & $38(11,0)$ \\
\hline It 6 & $112(32,4)$ & $78(22,5)$ & $77(22,3)$ & $29(8,4)$ & $25(7,2)$ & $25(7,2)$ \\
\hline It 7 & $208(60,3)$ & $80(23,2)$ & $35(10,1)$ & $5(1,4)$ & $4(1,2)$ & $13(3,8)$ \\
\hline It 8 & $268(77,9)$ & $35(10,2)$ & $23(6,7)$ & $10(2,9)$ & $4(1,2)$ & $4(1,2)$ \\
\hline It 9 & $88(25,4)$ & $70(20,2)$ & $93(26,9)$ & $41(11,8)$ & $25(7,2)$ & $29(8,4)$ \\
\hline It 10 & $186(53,8)$ & $53(15,3)$ & $56(16,2)$ & $13(3,8)$ & $22(6,4)$ & $16(4,6)$ \\
\hline It 11 & $202(58,4)$ & $75(21,7)$ & $43(12,4)$ & $14(4,0)$ & $6(1,7)$ & $6(1,7)$ \\
\hline It 12 & $47(13,6)$ & $88(25,4)$ & $106(30,6)$ & $47(13,6)$ & $33(9,5)$ & $25(7,2)$ \\
\hline It 13 & $238(68,8)$ & $63(18,2)$ & $24(6,9)$ & $8(2,3)$ & $7(2,0)$ & $6(1,7)$ \\
\hline It 14 & $113(32,7)$ & $81(23,4)$ & $71(20,5)$ & $31(9,0)$ & $19(5,5)$ & $31(9,0)$ \\
\hline It 15 & $111(32,1)$ & $77(22,3)$ & $69(19,9)$ & $32(9,2)$ & $32(9,2)$ & $25(7,2)$ \\
\hline It 16 & $167(48,3)$ & $45(13,0)$ & $57(16,5)$ & $37(10,7)$ & $21(6,1)$ & $19(5,5)$ \\
\hline It 17 & $125(36,1)$ & $64(18,5)$ & $62(17,9)$ & $32(9,2)$ & $38(11,0)$ & $25(7,2)$ \\
\hline It 18 & $251(72,8)$ & $51(14,8)$ & $30(8,7)$ & $4(1,2)$ & $5(1,4)$ & $5(1,2)$ \\
\hline It 19 & $204(59,0)$ & $53(15,3)$ & $50(14,5)$ & $15(4,3)$ & $15(4,3)$ & $9(2,6)$ \\
\hline It 20 & $112(32,6)$ & $109(31,7)$ & $70(20,3)$ & $28(8,1)$ & $19(5,5)$ & $6(1,7)$ \\
\hline It 21 & $149(43,2)$ & $56(16,2)$ & $57(16,5)$ & $39(11,3)$ & $25(7,2)$ & $19(5,5)$ \\
\hline It 22 & $161(46,5)$ & $60(17,3)$ & $38(11,0)$ & $26(7,5)$ & $31(9,0)$ & $30(8,7)$ \\
\hline It 23 & $126(36,4)$ & $63(18,2)$ & $61(17,6)$ & $42(12,1)$ & $26(7,5)$ & $28(8,1)$ \\
\hline It 24 & $98(28,3)$ & $62(17,9)$ & $71(20,5)$ & $53(15,3)$ & $33(9,5)$ & $29(8,4)$ \\
\hline It 25 & $245(71,2)$ & $44(12,8)$ & $24(7,0)$ & $12(3,5)$ & $10(2,9)$ & $9(2,6)$ \\
\hline It 26 & $322(93,1)$ & $10(2,9)$ & $8(2,3)$ & $4(1,2)$ & $1(0,3)$ & $1(0,3)$ \\
\hline It 27 & $268(77,5)$ & $43(12,4)$ & $19(5,5)$ & $9(2,6)$ & $5(1,4)$ & $2(0,6)$ \\
\hline It 28 & $123(35,5)$ & $78(22,5)$ & $62(17,9)$ & $27(7,8)$ & $32(9,2)$ & $24(6,9)$ \\
\hline It 29 & $117(33,8)$ & $110(31,8)$ & $58(16,8)$ & $31(9,0)$ & $17(4,9)$ & $13(3,8)$ \\
\hline It 30 & $121(35,0)$ & $82(23,7)$ & $66(19,1)$ & $31(9,0)$ & $25(7,2)$ & $21(6,1)$ \\
\hline It 31 & $124(35,9)$ & $88(25,5)$ & $55(15,9)$ & $29(8,4)$ & $27(7,8)$ & $22(6,4)$ \\
\hline It 32 & $324(93,9)$ & $10(2,9)$ & $5(1,4)$ & $2(0,6)$ & $2(0,6)$ & $2(0,6)$ \\
\hline It 33 & $106(30,6)$ & $100(28,9)$ & $77(22,3)$ & $33(9,5)$ & $17(4,9)$ & $13(3,8)$ \\
\hline It 34 & $46(13,3)$ & $36(10,4)$ & $61(17,6)$ & $60(17,3)$ & $58(16,8)$ & $85(24,6)$ \\
\hline
\end{tabular}


A validade convergente do BSQ foi adequada (VEM = 0,50; $C C=0,97$ ) e foi verificada adequada consistência interna $(a=0,97)$ do instrumento.

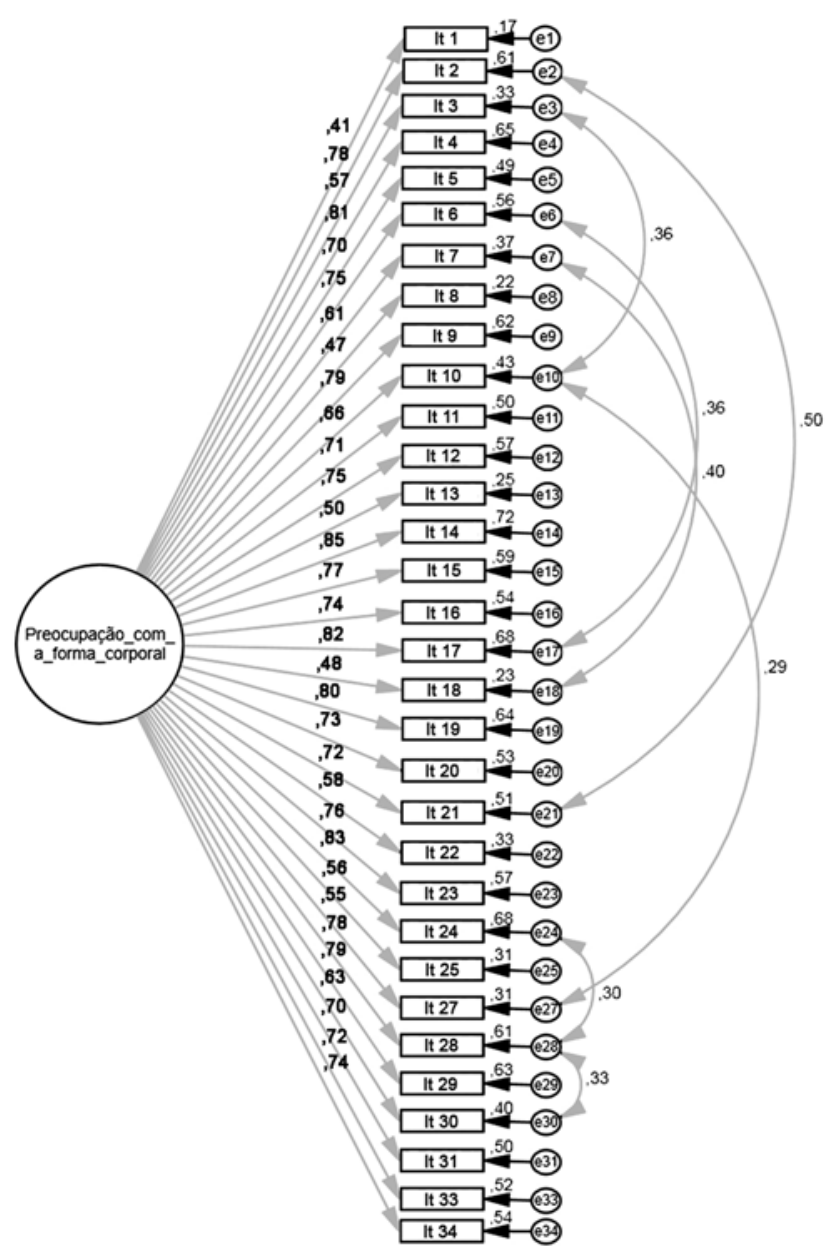

Figura 1. Modelo fatorial refinado do $B S Q$ ajustado à amostra de estudantes de Farmácia-Bioquímica $\left(\chi^{2} / g I=3,29 ; C F I=0,87\right.$; $\mathrm{NFI}=0,82 ; \mathrm{RMSEA}=0,08$. São Paulo, 2013).

Para a amostra de estudo, a preocupação com a forma do corpo pôde ser equacionada conforme apresentado a seguir:

BSQ $=0,016^{*} i t 1+0,019 * i t 2+0,015 * i t 3+0,008 * i t 4+0,026^{*} i t 5+0,012 * i t 6+0,011 * i t 7+0,011 *$ it $8+0,023 *$ it $9+0,018 *$ it $10+0,008 *$ it $11+0,008 *$ it $12+0,021 *$ it $13+0,028 *$ it $14+0,006 *$ it $15+$ $0,022^{*}$ it $16+0,017 *$ it $17+0,02^{*}$ it $18+0,033^{*}$ it $19+0,01^{*}$ it $20+0,021^{*}$ it $21+0,021^{*}$ it $22+0,008^{*}$ it $23+0,023^{*}$ it $24+0,011^{*}$ it $25+0,012^{*}$ it $27+0,011^{*}$ it $28+0,015^{*}$ it $29+0,023^{*}$ it $30+0,006^{*}$ it 31 $+0,015^{*} i \mathrm{it} 3+0,006^{*} \mathrm{it} 34$

A comparação do escore médio de preocupação com a forma do corpo segundo as variáveis de interesse encontrase na tabela 1.

Nota-se relação significativa entre a preocupação com a forma do corpo e o sexo, a avaliação em relação ao curso, o consumo de medicamentos por causa dos estudos, o pensamento em desistir do curso e o estado nutricional. As mulheres apresentam maior preocupação com a forma do corpo que os homens. Os indivíduos mais preocupados com a forma corporal foram aqueles com avaliação em relação ao curso pior do que a expectativa inicial, que consomem medicações por causa dos estudos, que já pensaram em desistir do curso e os que foram classificados em sobrepeso/ obesidade.

\section{DISCUSSÃO}

Destaca-se a contribuição deste estudo ao comparar a preocupação com a forma do corpo de estudantes de Farmácia-Bioquímica segundo características sociais, laborais e o estado nutricional. Cabe ressaltar, ainda, que neste trabalho, para cômputo do escore global de preocupação com a forma do corpo, utilizou-se o algoritmo respeitando as propriedades psicométricas do BSQ na amostra, aumentando, portanto, a precisão da estimativa realizada, sustentando, assim, a adequada validade e confiabilidade dos dados obtidos.

A análise fatorial confirmatória revelou que o BSQ apresentou adequada validade e confiabilidade, para a amostra, sem a presença dos itens 26 e 32 . Esses resultados corroboram a literatura22,24,32, reforçando que esses itens estão direcionados a comportamentos característicos de severas alterações relacionadas à alimentação e, portanto, não são capazes de contribuir, nessa escala, para a avaliação da preocupação com a forma do corpo em população normativa (Tabela 2).

A relação significativa verificada entre a preocupação com a forma do corpo e as variáveis sexo, avaliação em relação ao curso, uso de medicamentos por causa dos estudos, pensamento em desistir do curso e estado nutricional tem sido explicada em estudos anteriores.

A maior preocupação com a forma do corpo é encontrada entre as estudantes do sexo feminino ${ }^{19,29,31,41}$. De acordo com Miranda et al. ${ }^{41}$ e Branco et al. ${ }^{19}$, as mulheres apresentam maiores preocupações com a aparência física, considerando as fortes pressões estabelecidas pela cultura e pela sociedade em que vivem, sendo condicionadas a manter o corpo no padrão desejável "magro", apontado como adequado. Apesar de os homens também apresentarem preocupações relacionadas ao corpo, estas estão voltadas a aspectos mais específicos como ganho de massa muscular, calvície e virilidade ${ }^{8,42,43}$. Essas características peculiares ao sexo masculino não são abordadas nos itens da escala BSQ, assim esse fato pode justificar a baixa preocupação com a forma do corpo encontrada nesse estudo nesses indivíduos e alertar para o fato de que instrumentos que captam as especificidades dessa população poderiam capturar de maneira mais acurada a preocupação dos homens quanto ao corpo.

A maior preocupação com a forma do corpo dos indivíduos cuja avaliação do curso foi pior que as expectativas iniciais, que usam medicamentos por causa dos estudos 
e que já pensaram em desistir do curso sugere que pode existir tensão e ansiedade nesses estudantes no ambiente universitário e esses aspectos podem estar interferindo nas suas percepções em relação ao corpo. Yanover e Thompson ${ }^{44}$ destacam que as fortes pressões estabelecidas na universidade estão diretamente relacionadas às preocupações voltadas ao corpo e ainda acrescentam que, quanto maior a apreensão e/ou insatisfação dos jovens com o curso escoIhido, maiores são as preocupações com a aparência física.

Outro resultado encontrado nesse estudo, que é sustentando pela literatura ${ }^{41,45,46}$, é a relação significativa entre a preocupação com a forma do corpo e o estado nutricional. Ferrari et al. ${ }^{45}$ destacam que os indivíduos classificados com sobrepeso/obesidade apresentam maiores preocupações quanto à aparência física. Outros estudos ${ }^{19,41}$ ainda acrescentam que, quanto maior o peso corporal dos indivíduos, maiores são as preocupações em relação ao corpo.

Esse estudo buscou investigar características importantes relacionadas à preocupação com a forma do corpo de estudantes de Farmácia-Bioquímica. Foram identificadas variáveis significativas que podem ser utilizadas em protocolos educativo-preventivos para minimizar os dados decorrentes da exacerbada preocupação com o corpo e também auxiliar na área de pesquisa em imagem corporal, destacando pontos que podem ser investigados para o rastreamento de percepção alterada em relação ao corpo.

\section{CONCLUSÃO}

A preocupação dos estudantes universitários com a forma do corpo esteve relacionada com o sexo, a avaliação em relação ao curso, a ingestão de medicamentos por causa dos estudos, o pensamento em desistir do curso e o estado nutricional.

\section{CONTRIBUIÇÕES INDIVIDUAIS}

Todos os autores contribuíram significativamente na concepção e desenho do estudo deste trabalho. Os autores participaram efetivamente no delineamento e na confecção do projeto de pesquisa, na coleta, análise e interpretação dos dados e também na elaboração intelectual do artigo científico, sendo de comum acordo entre todos a aprovação da versão final enviada para publicação.

\section{CONFLITOS DE INTERESSE}

Nenhum dos autores apresenta qualquer potencial de conflito de interesse.

\section{AGRADECIMENTOS}

À Fundação de Amparo à Pesquisa do Estado de São Paulo (Fapesp), pelo financiamento do estudo (Grant\# 2013/055099, São Paulo Research Foundation - Fapesp).

\section{REFERÊNCIAS}

1. Campana ANNB, Tavares MCGCF, Garcia Júnior C. Preocupação e insatisfação com 0 corpo, checagem e evitação corporal em pessoas com transtornos alimentares. Paidéia. 2012;22(53):375-81.

2. Cash TF, Fleming EC. The impact of body image experiences: development of the body image quality of life inventory. Int J Eat Disord. 2002;31(4):455-60.

3. Thompson JK. The (mis)measurement of body image: ten strategies to improve assessment for applied and research purposes. Body Image. 2004;1(1):7-14.

4. Sarwer DB, Cash TF. Body image: interfacing behavioral and medical sciences. Aesthet Surg J. 2008;28(3):357-8.

5. Campana ANNB, Tavares MCGCF. Avaliação da imagem corporal: instrumentos e diretrizes para a pesquisa. São Paulo: Phorte Editora; 2009. 232p.

6. Akdemir A, Inandi T, Akbas D, Kahilogullari AK, Eren M, Canpolat BI. Validity and reliability of a Turkish version of the Body Shape Questionnaire among female high school students: preliminary examination. Eur Eat Disord Rev. 2012;20(1):e114-5.

7. Baranowksi MJ, Jorga J, Djordjevic I, Jelena, Hetherington MM. Evaluation of adolescent body satisfaction and associated eating disorder pathology in two communities. Eur Eat Disord Rev. 2003;11:478-95.

8. McFarland MB, Petrie TA. Male body satisfaction: factorial and construct validity of the Body Parts Satisfaction Scale for Men. J Couns Psychol. 2012;59(2):329-37.

9. Najam N, Ashfaq H. Gender differences in physical fitness, body shape satisfaction, and body figure preferences. Pakistan Journal of Psychological Research. 2012;27(2):187-200.

10. Costa LCF, Vasconcelos FAG. Influence of socioeconomic, behavioral and nutritional factors on dissatisfaction with body image among female university students in Florianopolis, SC. Rev Bras Epidemiol. 2010;13(4):665-76.

11. Bosi MLM, Luiz RR, Morgado CMC, Costa MLS, Carvalho RJ. Autopercepção da imagem corporal entre estudantes de nutrição: um estudo no município do Rio de Janeiro. J Bras Psiquiatr. 2006;55(2):108-13.

12. Conti MA, Cordás TA, Latorre MRDO. A study of the validity and reliability of the Brazilian version of the Body Shape Questionnaire (BSQ) among adolescents. Rev Bras Saúde Matern Infant. 2009;9(3):331-8

13. Silva JD, Silva ABJ, Oliveira AVK, Nemer ASA. Influência do estado nutricional no risco para transtornos alimentares em estudantes de nutrição. Ciênc Saúde Coletiva. 2012;17(12):3399-406.

14. Silva WR, Dias JCR, Maroco J, Campos JADB. Confirmatory factor analysis of different versions of the Body Shape Questionnaire applied to Brazilian university students. Body Image. Body Image. 2014;11(4):384-90.

15. Garner DM, Olmsted MP. Manual for the Eating Disorder Inventory (EDI). Odessa, FI: Psychological Assessment Resources; 1984.

16. Williamson DA, Davis CJ, Bennett SM, Goreczny AJ, Gleaves DH. Development of a simple procedure for assessing body image disturbances. Behav Assess. 1989;11(4):443-6.

17. Rosen JC, Srebnik D, Saltzberg E, Wendt S. Development of a body image avoidance questionnaire. Psychol Assess J Consult Clin Psychol. 1991;3(1):32-7.

18. Cooper PJ, Taylor MJ, Cooper Z, Fairburn CG. The development and validation of the Body Shape Questionnaire. Intl J Eat Disord. 1987;6(4):485-94.

19. Branco LM, Hilário MOE, Cintra IP. Percepção e satisfação corporal em adolescentes e a relação com seu estado nutricional. Rev Psiq Clín. 2006;33(6):292-6.

20. Di Pietro M, Silveira DX. Internal validity, dimensionality and performance of the Body Shape Questionnaire in a group of Brazilian college students. Rev Bras Psiquiatr. 2009;31(1):21-4

21. Kakeshita IS, Almeida SS. Relação entre índice de massa corporal e a percepção da autoimagem em universitários. Rev Saúde Pública. 2006;40(3):497-504. 
22. Evans C, Dolan B. Body Shape Questionnaire: derivation of shortened "alternate forms". Int J Eat Disord. 1993;13(3):315-21.

23. Ghaderi A, Scott B. The reliability and validity of the Swedish version of the Body Shape Questionnaire. Scand J Psychol. 2004;45(4):319-24.

24. Pimenta F, Leal I, Maroco J, Rosa B. Validação do Body Shape Questionnaire (BSQ) numa amostra de mulheres de meia-idade. Actas do $9^{\circ}$ Congresso Nacional de Psicologia da Saúde; Aveiro, Portugal: Placebo; 2012. p. 1139-44.

25. Pook M, Tuschen-Caffier B, Brähler E. Evaluation and comparison of different versions of the Body Shape Questionnaire. Psychiatry Res. 2008:158:67-73.

26. Bosi MLM, Luiz RR, Uchimura KY, Oliveira FP. Comportamento alimentar e imagem corporal entre estudantes de educação física. J Bras Psiquiatr. 2008;27(1):28-33.

27. Sepulveda AR, Carrobles JA, Gandarilhas AM. Gender, school and academic year differences among Spanish university students at high-risk for developing an eating disorder: an epidemiologic study. BMC Public Health. 2008;8:102.

28. Maruf FA, Akinpelu A0, Udoji NV. Differential perceptions of body image and body weight among adults of different socioeconomic status in a sub-urban population. J Biosoc Sci. 2014;46(3):279-93.

29. Martins CR, Gordia AP, Silva DAS, Quadros TMB, Ferrari EP, Teixeira DM, et al. Insatisfação com a imagem corporal e fatores associados em universitários. Estud Psicol. 2012;17(2):241-6.

30. Garcia L, Milagres OG, Mourão L, Assis M, Palma A. Autopercepção da imagem corporal em estudantes de Educação Física e Medicina. Revista Brasileira de Atividade Física \& Saúde. 2011;16(1):25-30.

31. Legnani RFS, Legnani E, Pereira EF, Gasparotto GS, Vieira LF, Campos W. Transtornos alimentares e imagem corporal em acadêmicos de Educação Física. Motricidade. 2012;18(1):84-91.

32. Silva WR, Dias JCR, Maroco J, Campos JADB. Comparison of different versions of the Scale of Concern with body shape applied to university students. Fapesp: processo: 2012/13475-4 e 2013/02678-4. 2013.

33. Hair JF, Black WC, Babin B, Anderson RE, Tatham RL. Multivariate data analysis. 6th ed. Upper Saddle River, NJ: Pearson Prentice Hall; 2005. 928p.
34. WHO. Obesity: preventing and managing the global epidemic. Geneva: World Health Organization: WHO; Technical Report Series; 2000.

35. ABEP. Associação Brasileira de Empresas de Pesquisa. Critério de Classificação Econômica Brasil 2008. Disponível em: <http://abep.org/codigosguias/Critério_Brasil_2008.pdf>. Acesso em: 7 mar. 2013.

36. Cooper PJ, Taylor MJ, Cooper Z, Fairburn CG. The development and validation of the Body Shape Questionnaire. Int J Eat Disord. 1987;6(4):485-94.

37. Maroco J. Análise de equações estruturais: fundamentos teóricos, software \& aplicações. Lisboa: ReportNumber; 2014. 400p.

38. Fornell C, Larcker DF. Evaluating structural equation models with unobservable variables and measurement error. J Marketing Res. 1981;18(1):39-50.

39. Maroco J, Garcia-Marques T. Qual a fiabilidade do alfa de Cronbach? Questões antigas e soluções modernas? Laboratório de Psicologia. 2006;4(1):65-90.

40. Campos JADB, Bonafé FSS, Dovigo LN, Maroco J. Avaliação psicométrica da escala de atitudes em relação à estatística. Rev Bras Biom. 2013;31(2):327-37.

41. Miranda VPN, Filgueiras JF, Neves CM, Teixeira PC, Ferreira MEC. Insatisfação corporal em universitários de diferentes áreas de conhecimento. Rev Bras Psiquiatr. 2012;61(1):25-32.

42. Fisher $E$, Dunn $M$, Thompson JK. Social comparison and body image: an investigation of body comparison processes using multidimensional scaling. I Soc Clin Psychol. 2002:21(5):566-79.

43. McCreary DR, Sasse DK. An exploration of the drive for muscularity in adolescent boys and girls. J Am Coll Health. 2000;48(6):297-304

44. Yanover T, Thompson J. Eating problems, body image disturbances, and academic achievement: preliminary evaluation of the eating and body image disturbances academic interference scale. Int J Eat Disord. 2008:41(2):184-7.

45. Ferrari EP, Gordia AP, Martins CR, Silva DA, Quadros TM, Petroski EL. Insatisfação com a imagem corporal e relação com o nível de atividade física e estado nutricional em universitários. Motricidade. 2012;8(3):52-8.

46. Laus MF, Moreira RCM, Costa TMB. Diferenças na percepção da imagem corporal, no comportamento alimentar e no estado nutricional de universitárias das áreas de saúde e humanas. Rev Psiquiatr RS. 2009;31(3):192-6. 